\title{
Monitoreo neurofisiológico intraoperatorio en traumatismo raquimedular cervical y columna cervical compleja: Recomendaciones desde una visión dual basada en experiencia personal
}

\author{
Intraoperative neurophysiological monitoring during traumatic \\ cervical spinal cord injury and complex cervical spine: \\ Recommendations from a double vision based on personal \\ experience
}

\author{
Juan P. Cabrera Cousiño' \\ ${ }^{1}$ Servicio de Neurocirugía, Hospital Clínico Regional de Concepción, Universidad de Concepción. Concepción, Chile.
}

\section{Resumen}

Introducción: La cirugía de columna cervical compleja, traumática o de otras causas, puede determinar un nuevo déficit neurológico incluso en manos expertas, riesgo que comienza desde la posición quirúrgica, y más aún durante instrumentación o maniobras de corrección. Sin embargo, el uso de Monitoreo Neurofisiológico Intraoperatorio (MIO) entrega información funcional en tiempo real que pudiera definir pasos quirúrgicos, y consecuentemente, mejor pronóstico neurológico. Objetivo: Demostrar la necesidad de utilizar MIO en cirugías complejas de columna cervical, entregando recomendaciones prácticas para estas cirugías desde una visión dual. Material y Método: Estudio prospectivamente recolectado y retrospectivamente analizado, del conjunto de pacientes intervenidos de columna cervical y cervicotorácica compleja, en quienes fue necesario intervenir quirúrgicamente bajo MIO, durante período 2013-2018, con énfasis en pacientes intervenidos y monitorizados por el autor. Se excluyen pacientes intervenidos de disco cervical sin mielopatía. Resultados: Durante este período, un total de 1.305 pacientes fueron intervenidos bajo MIO, de los cuales 217 corresponden a columna cervical, y 142 a columna cervical compleja. De ellos, en 55 el autor participa como asistente quirúrgico y $\mathrm{MIO}$, y en 37 como primer cirujano + MIO. De estos 37 pacientes, la causa traumática es la que predomina, seguido por degenerativo. Tres pacientes presentan caída significativa de potenciales transitoria, que responden a maniobras de reanimación, evolucionando sin deterioro neurológico. Conclusiones: EI MIO, en columna cervical compleja, entrega información y seguridad no evaluable a través de ninguna otra herramienta disponible en Neurocirugía, determinando cambios quirúrgicos intraoperatorios y como curva de aprendizaje que favorecen significativamente los resultados.

Palabras clave: Columna cervical, Monitoreo Neurofisiológico Intraoperatorio, Traumatismo raquimedular, Cirugía columna compleja.

\section{Abstract}

Introduction: Complex cervical spine surgery, traumatic or of other causes, can determine a new neurological deficit even in expert hands, risk that starts from the surgical position, and even more during instrumentation or correction maneuvers. However, during these surgeries the Intraoperative Neurophysiological Monitoring (IOM) provides real-time functional information that could define surgical steps, and consequently, better neurological outcome. Objective: To demonstrate the need to use IOM in complex cervical spine surgeries, delivering practical recommendations

\section{Correspondencia a:}

jucabrera@udec.cl 
for these surgeries since their use. Material and Method: A prospectively collected and retrospectively analyzed study of the group of patients undergoing cervical and complex cervicothoracic spinal surgery, in whom it was necessary to undergo surgery under IOM, during the period 2013-2018, with emphasis on patients operated on and monitored by the author. We exclude patients who underwent a cervical disc without myelopathy. Results: During this period, a total of 1,305 patients underwent surgery under IOM, of which 217 corresponded to the cervical spine, and 142 to a complex cervical spine. Of these, in 55 the author participates as a surgical assistant and MIO, and in 37 as the first surgeon + MIO. Of these 37 patients, the traumatic cause predominates, followed by degenerative. Three patients presented significant and fall of potentials, which respond to resuscitation maneuvers, evolving without neurological deterioration. Conclusions: The IOM, in a complex cervical spine, provides information and safety that cannot be evaluated through any other tool available in Neurosurgery, determining intraoperative surgical changes and learning curve that significantly favor the surgical results.

Key words: Cervical spine, Intraoperative neurophysiological monitoring, Spinal column trauma, Complex spine surgery.

\section{Introducción}

El traumatismo de columna constituye una causa importante de morbimortalidad en la actualidad, ocasionando discapacidad en población joven y laboralmente activa, con consecuencias negativas a nivel personal, familiar, social y económico ${ }^{1}$. En casos de lesión inicial incompleta, su recuperación depende significativamente de los factores de la injuria como severidad, mecanismo y nivel lesional, pero no está asociado con el tipo de tratamiento realizado o país de origen².

La cirugía electiva de columna cervical simple, esto es, discos blandos únicos en canal no estrecho y en paciente sin comorbilidades, o foraminotomía posterior unilateral, si bien no están exentas de riesgo, la probabilidad de daño neurológico es bajo, y cuando sucede, es transitoria ${ }^{3}$. Al contrario, pacientes con comorbilidades sometidos a cirugía compleja de columna cervical, que requieran instrumentaciones mas extensas, descompresión directa o indirecta amplia, abordaje anterior y posterior, mielorradiculopatía pre-existente, correcciones de balance cervical, o reducciones en contecto traumático, degenerativo o tumoral, y muy en especial al existir déficit neurológico parcial, la probabilidad de deterioro permanente es una posibilidad real.

La variedad de presentación de un nuevo déficit en este contexto es amplia, pudiendo diferenciarlos para fines semánticos como radicular, medular, mixto u otro. Origen radicular destaca radiculopatía $\mathrm{C5}$, especialmente en vía posterior pudiendo llegar al 13\%, paresia C8-T1 y Síndrome Parsonnage-Turner; medular en sitio lesional, pudiendo ascender por edema y/o siringomielia; mixto combinación segmentaria de las previas; otras como nervio laríngeo recurrente en vías anteriores a columna cervical subaxial baja y con mayor incidencia al realizar corpectomía, y Síndrome de Horner en aquelos casos que requieran acceder lateral al músculo longus colli, para esqueletización de arteria vertebral vía anterior en contexto tumoral, deformidades rígidas $u$ otras ${ }^{3}$.

El Monitoreo Neurofisiológico Intraoperatorio es actualmente el estándar de cuidado en cirugía de columna de deformidades, traumática y degenerativa, en especial en pacientes en quienes se planifica instrumentación y/o descompresión como parte del plan quirúrgico. Por otra parte, las consecuencias de un traumatismo raquimedular cervical son muchas veces devastadoras, y la cirugía un desafío incluso en manos expertas.

Por otra parte, monitorizar estos casos, en especial aquellos con mielopatía de cualquier origen instaurada, también en un desafío, que comienza dede la posición quirúrgica en adelante, requiriendo técnicas de estimulación y registro diferentes, y en que la interpretación de ellas es crucial y determinante en maniobras quirúrgicas en tiempo real que definen pronóstico funcional. El MIO brinda beneficios en este respecto a pacientes indemnes, con déficit parcial, incluso a pacientes con profundo déficit neurológico preoperatorio ${ }^{4}$.

El objetivo es demostrar la necesidad de utilizar MIO en cirugías complejas de columna cervical, entregando recomendaciones prácticas para estas cirugías desde una visión dual, neuroquirúrgica y de $\mathrm{MIO}$, en base a experiencia personal. Para lo anterior, se consideran entendidas las clasificaciones de fracturas, cuadros clínicos, escalas complementarias, estudio de balance cervical, etc. que no representan el objetivo del trabajo actual.

\section{Material y Método}

Estudio prospectivamente recolectado y retrospectivamente analizado, del conjunto de pacientes intervenidos de columna cervical y cervicotorácica compleja, en quienes fue necesario la utilización de MIO, durante el período 20132018, con especial énfasis en pacientes intervenidos y monitorizados por el autor.

Se excluyen pacientes de columna cervical disco simple sin mielopatía (incluso monitorizados), además de descompresiones $\mathrm{C} 0-\mathrm{C} 1$ en Chiari o acondroplasias. Se excluyen pacientes con deformidad cervical compensatoria a desbalance sagital y/o coronal toracolumbar operados, y se excluyen pacientes con escaso o pérdida de seguimiento.

\section{Técnica anestésica}

Para fines de MIO, pacientes fueron intervenidos con régimen de Anestesia Total Intravenosa (TIVA) salvo excepciones o no disponibilidad. Se mantiene con bomba infusión 
continua propofol + remifentail, con índice biespectral entre 30-45. En aquellos casos que no fue posible contar con TIVA, Sevofluorano o Desfluorano fue el esquema de elección, manteniendo CAM menor a 1.0, idealmente cercana a 0.6. Bloqueo neuromuscular de vida media corta o intermedia es infundido durante inducción anestésica en caso de vía aérea difícil o preferencia de anestesista, y revertido con Sugammadex en caso de disponibilidad. Para aquellos pacientes que requieran potencial basal en supino, bloqueo neuromuscular fue evitado, revertido o esperado hasta respuestas confiables y reproductibles, evaluado a través de TOF, garantizando desbloqueo de placa neuromuscular. Bloqueo de mordida con gasas intraorales en todos los pacientes para evitar corte-laceración de lengua, daño de tubo endotraqueal u otros. Línea arterial en todos estos casos complejos, procurando mantener PAM adecuadas según etapa quirúrgica y eventos intraoperatorios.

\section{Técnica de MIO}

Variable según paciente. Se cuenta con máquina NIMEclipse (Medtronic) de 32 canales, con potenciales evocados motores transcraneales y somatosensoriales tronculares, electromiografía corriendo libre, electromiografía evocada, estimulación de tornillos transpediculares, TOF, reflejos, Onda-D en casos determinados. En casos de alto riesgo, se toma potenciales evocados basales en posición supino y columna cervical neutra, identificando latencias y amplitudes, morfología y número de fases según corresponda. Registro con agujas dobles subdermales de $13 \mathrm{~mm}$ insertas en músculos diana según caso, abarcando las 4 extremidades. Montaje craneal según Sistema Internacional 10-20, con sacacorchos en cuero cabelludo. En aquellos casos en que no se pudo obtener potenciales confiables distales a sitio lesional o en riesgo, técnicas de estimulación y registro diferentes a las tradicionales fueron utilizadas. Se cuenta con Tecnólogo Médico entrenado por el autor, y pantalla para cirujano simultánea, a modo de en todo momento contar con doble evaluación. No se profundiza en parámetros electrofisiológicos de cada modalidad, a modo de simplicar la compresión.

\section{Técnica quirúrgica}

Dependiente del caso y patología, condiciones del paciente. En casos de mielopatía cervico-artrósica 1 nivel, disquectomía + cage fue preferido; en casos de mas de 1 nivel de estenorraquis significativa, con conflicto de espacio mas amplio que solo a nivel discal, vía posterior fue de elección. En casos traumáticos, la variedad es amplia: casos con luxación facetaria leve unilateral y listesis grado I, cage + placa; luxofractura facetaria bilateral, vía posterior de reducción, estabilización y descompresión en casos seleccionados, seguido por vía anterior cage + placa; algunas mielopatías compresivas y Sd. Centromedular post-traumático, descompresión mielorradicular directa y fijación, laminoplastía en pacientes de muy alto riesgo o contraindicación relativa de instrumentación, descompresiva sola en primeros años y actualmente contraindicada. Patología tumoral osteolítica o infecciosa con inestabilidad, deformidad cifótica cualquier origen, estabilización circunferencial y descompresión directa o indirecta, comenzando vía anterior corporectomía 2-3 niveles + placa anterior seguido de fijación posterior larga en mismo tiempo.

Instrumentación a columna cervical posterior subaxial C3-C6 con tornillos a masas laterales modo "free-hand" y técnica Magerl modificada, C7-T2 tornillos transpediculares bajo radioscopía AP exclusiva o "free-hand" menos ocasionalmente. Instrumental de variadas compañías, pero preferencia sistema Mini-Mesa (K2M) o sistema empresa Synthes. Vías anteriores con corpectomía canastillo expandible + placa empresa Globus Medical o Synthes. Cage de PEEK + placa anterior diversas empresas, no habiendo utilizado cage autoestables para patología traumática.

Pacientes intervenidos son sometidos a tomografía computada de columna cervical o cervicodorsal el día siguiente a intervención quirúrgica, salvo nuevo deterioro neurológico, evaluando posición de instrumentación, grado de descompresión directa o indirecta, y parámetros de balance cervical cuando se intentaron modificar, tales como lordosis cervical C2-C7, cSVA (saggital vertical axis cervical), TIA (thoracic inlet angle), T1 slope, entre otros. Seguimiento postoperatorio y ambulatorio a todos, evaluando fusión al menos a 6 meses e idealmente 2 años, y descarte activo de complicaciones tardías como pseudoartrosis, extrusión material de osteosíntesis, cifosis de la unión proximal (PJK), discopatía del segmento adyacente o fractura segmento adyacente, entre otras.

\section{Resultados}

Durante este período, se realizaron 1.305 intervenciones quirúrgicas bajo Monitorización Neurofisiológica Intraoperatoria, de los cuales 998 corresponden a patología de columna de cualquier etiología (76,5\%). De estas, 217 corresponden a patología de columna cervical o cervicotorácica $(21,7 \%$ de las columnas), siendo 142 casos patología de columna cervical compleja (14,2\%).

Dentro del grupo de patología de columna cervical compleja monitorizada, el autor participó como asistente quirúrgico y MIO paralelamente en 55 casos $(38,7 \%$ de las cervicales complejas), y en 37 casos como primer cirujano + MIO (26,1\%). Existe un 35,2\% de las columnas cervicales monitorizadas en que el autor solamente realiza $\mathrm{MIO}$, sin rol quirúrgico como cirujano ni asistente.

Dentro del grupo de asistente $+\mathrm{MIO}$, destaca 2 niños intervenidos por equipo de NC pediátrica con asistencia columna, en quienes se extirpó tumores osteolíticos amplios extradural, con instrumentación corta dada fascetectomía amplia bilateral. En seguimiento no han desarrollado deformidad cifótica post-laminectomía ni cifosis de la unión proximal (PJK). Resto de los pacientes son mayores de 18 años, rango hasta 83 años.

Las etiologías más frecuentemente intervenidas fueron patología traumática, degenerativa, tumoral, deformidad $2^{\mathrm{a}}$, deformidad 1aㅡ , infeccioso. De degenerativo, se optó por vía posterior en la mayoría al existir estenorraquis multinivel con mielopatía no resolvible en su totalidad a través del tratamiento de discos múltiples. En HNP disco-osteofítica, se prefirió vía anterior. En contexto traumático, domina luxofractura facetaria uni o bilateral cuando es precoz, de ellos, si tiene listesis grado I y luxación unilateral, reducción cerrada + ACDF/placa fue de elección o simplemente ACDF/placa que 
reduce luxación a través de pequeña fractura fragmento más apical de fasceta inferior luxada. Existen 2 pacientes quienes no fueron pesquizados a través del Servicio de Urgencia y desarrollaron una listesis asociado a cifosis angular, con diástasis facetaria bilateral, que fueron resueltos a través de ACDF/placa y $360^{\circ}$ otro. En luxaciones facetarias más severas, con listesis grado III o mayor, vía posterior de inicio fue prefererida (2 casos), realizando facetectomía amplia y alineación, fijación larga 3 segmentos proximal y 3 segmentos distal, o 2 distales transpediculares y 3 proximales. No se detuvo instrumentación en $\mathrm{C} 7$ en constructos largos, se llegó hasta $\mathrm{T} 1$ o T2 si T1 slope es pronunciado $\left(>30^{\circ}\right)$.

Existen casos mixtos, quienes son portadores de estenorraquis multinivel cervico-artrósica, y frente a traumatismo, desarrollaron Sd. Centromedular, y cuadro clínico compatible de tetraparesia predominio extremidades superiores. En ellos, descompresión mielorradicular amplia directa e instrumentación fue preferida. Pacientes con mala calidad ósea por enfermedad metastásica, osteoporosis, o pacientes con espondilitis anquilosante o HEDI fracturados, instrumentaciones largas fueron de regla.

Existen tres casos quienes presentan caída de potenciales significativa aunque transitoria, 2 en contexto de corrección de cifosis con canastillo expandible y 1 durante descompresión. Tras maniobras de reanimación y aumento PAM, avanzan a mejoría progresiva y canastillo se deja con menor grado de distracción. En contexto de descompresión, se amplió descompresiva a craneal y caudal además de maniobras anestésicas, con barra de estabilización transitoria in situ. En esta serie, no hay caída de potenciales secundaria a malposición de instrumentación. En grupo de asistente +
MIO, 3 pacientes presentaron caída, 2 transitoria durante descompresión posterior, y 1 definitiva con repercusión clínica: caso realizado 2013 con descompresión vía posterior pura. Dos pacientes tuvieron caída significativa de potenciales de origen postural (de supino a prono), y 3 pacientes plexopatía braquial postural, quienes al corregir posición quirúrgica recuperaron y sin repercusión clínica.

Se grafica la experiencia personal en base a casos representativos de las distintas etiologías, todos los cuales fueron realizadas bajo esta modalidad dual: cirujano principal + neuromonitoreo.

\section{Casos representativos}

\section{Degenerativo}

Paciente de 71 años, sexo femenino, HTA en tratamiento, cuadro de cervicalgia larga data asociado a impotencia funcional. Evoluciona con tetraparesia M4 predominio extremidades superiores, logrando deambular y sin conflicto esfinteriano. Se estudia con TC + RNM columna cervical que evidencia estenorraquis degenerativa multisegmentaria, con signos de mielopatía en secuencia T2 (Figura 1A). Dada compresión multinivel con conflicto de espacio mayor a lo que implica altura disco cervical, se define realizar descompresión + fijación vía posterior bajo MIO (Figura 1B). Evoluciona favorablemente y sin complicaciones, con RNM postoperatoria diferida que evidencia aumento ostensible del diámetro canal medular (Figura 1C), y TC columna alejado que confirma fusión facetaria niveles intervenidos con respeto de segmentos adyacentes craneal y caudal (Figuras 1D y 1E).
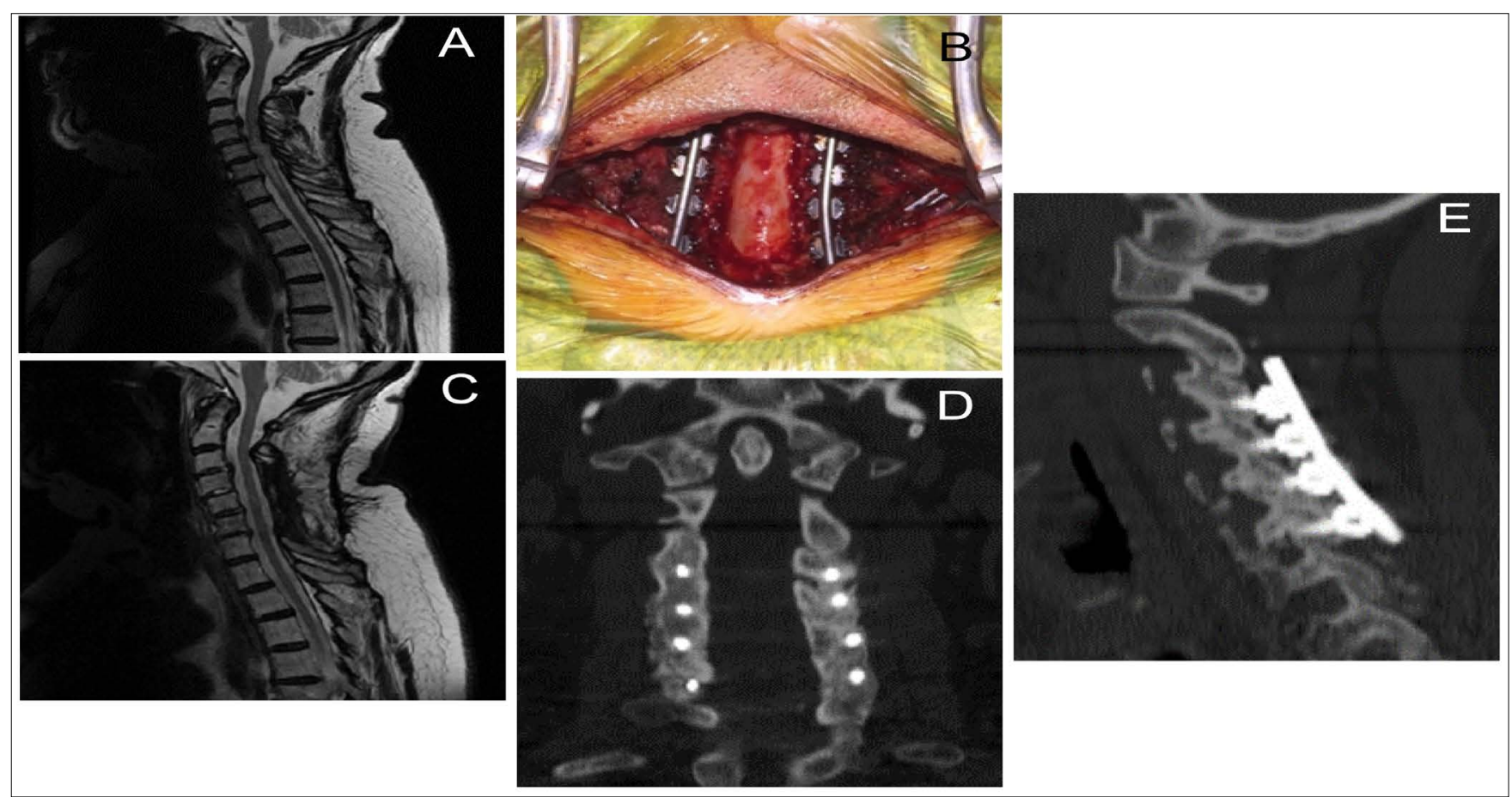

Figura 1. Caso degenerativo. A) RNM columna cervical evidenciando estenorraquis cervical multisegmentaria y mielopatía; B) Imagen intraopereatoria de descompresión + fijación vía posterior; C) RNM columna cervical postoperatoria diferida que demuestra adecuada descompresión medular; D) TC coronal alejado que demuestra fusión facetaria; E) TC sagital alejado que confirma fusión con sistema osteosíntesis in situ. 


\section{Traumático}

Paciente masculino de 19 años, sano previamente, sufre accidente de tránsito en motocicleta con casco de protección, resultando en traumatismo raquimedular cervicodorsal. Ingresa con collar cervical y neurológicamente indemne. Se estudia con TC columna total que demuestra fractura C2 tipo II odontoides (Figura 2A) y masa lateral izq. C1 flotante (Figura 2B), fractura corporal C7 (Figura 2A) y aplastamiento T4 en contexto escoliosis dextroconvexa 24 $\mathrm{AC}$ (Figura $2 \mathrm{C}$ ). Se decide tratamiento quirúrgico de todas las fracturas en un tiempo quirúrgico (Figura 2D), procedimiento sin incidentes bajo MIO, utilizando musculatura de lengua como registro proximal a C1-C2 (Figura 2E). Evoluciona favorablemente, levantándose y caminando al día siguiente, alta hospitalaria precoz.

\section{Tumoral}

Paciente femenino de 54 años, sin antecedentes mórbidos, debuta con cáncer gástrico a través de cervicalgia intensa con contractura muscular, indemne neurológicamente. Se estudia con TC (Figura 3A) + RNM (Figura 3B) columna cervicodorsal y estudio primario, evidenciando ganglios mediastínicos, y lesiones osteolíticas C6-C7-T1 con criterios de inestabilidad. Escalas compatibles apuntan a criterio quirúrgico, y sobrevida global esperada mayor a 6 meses. Se define intervención quirúrgica $360^{\circ}$ comenzando vía posterior, fijación C4-T4 y posteriormente canastillo C6-C7-T1 + placa anterior C5-T2 bajo Monitorización Neurofisiológica Intraoperatoria. Estimulación de tornillos torácicos altos técnica multipulso en umbral de seguridad (Figura 3C). Evolución postoperatoria favorable, con radiografía (Figura 3D) y TC que demuestra constructo in situ (Figuras 3E y 3F). Paciente fallece a los 11 meses habiendo presentado mejoría en calidad de vida.

\section{Infeccioso}

Paciente masculino de 59 años, $\mathrm{OH}(+)$ crónico, cuadro de fiebre origen desconocido y lumbago, que se estudia con RNM columna total evidenciando espondildiscitis L2-L3 y absceso del psoas izq. Se definió manejo médico antibiótico. Evoluciona con cervicalgia asociado a hipoestesia ambas EE.SS. Se estudia con TC + RNM columna cervical que evidencia espondilodiscitis C6-C7 sin signos de inestabilidad. Se mantuvo con tratamiento antibiótico, pero frente a persistencia sintomatología a pesar de tratamiento médico bien llevado con germen aislado, se vuelve a estudiar demostrando deformidad cifótica cervical C6-C7 con colapso cuerpo vertebral C6 y estenorraquis segmentaria (Figura 4A). Se define tratamiento quirúrgico, realizándose corporectomía C6-C7 + canastillo expandible y placa anterior C5-T1, y vía posterior C5-T2 bajo MIO, con potenciales somatosensoriales (Figura $4 \mathrm{~B})$ y motores. Evoluciona favorablemente y sin incidentes, con mejoría ostensible de SVA C2-C7 y lordosis cervical (Figura 4C), descompresión indirecta, indemne neurológica-

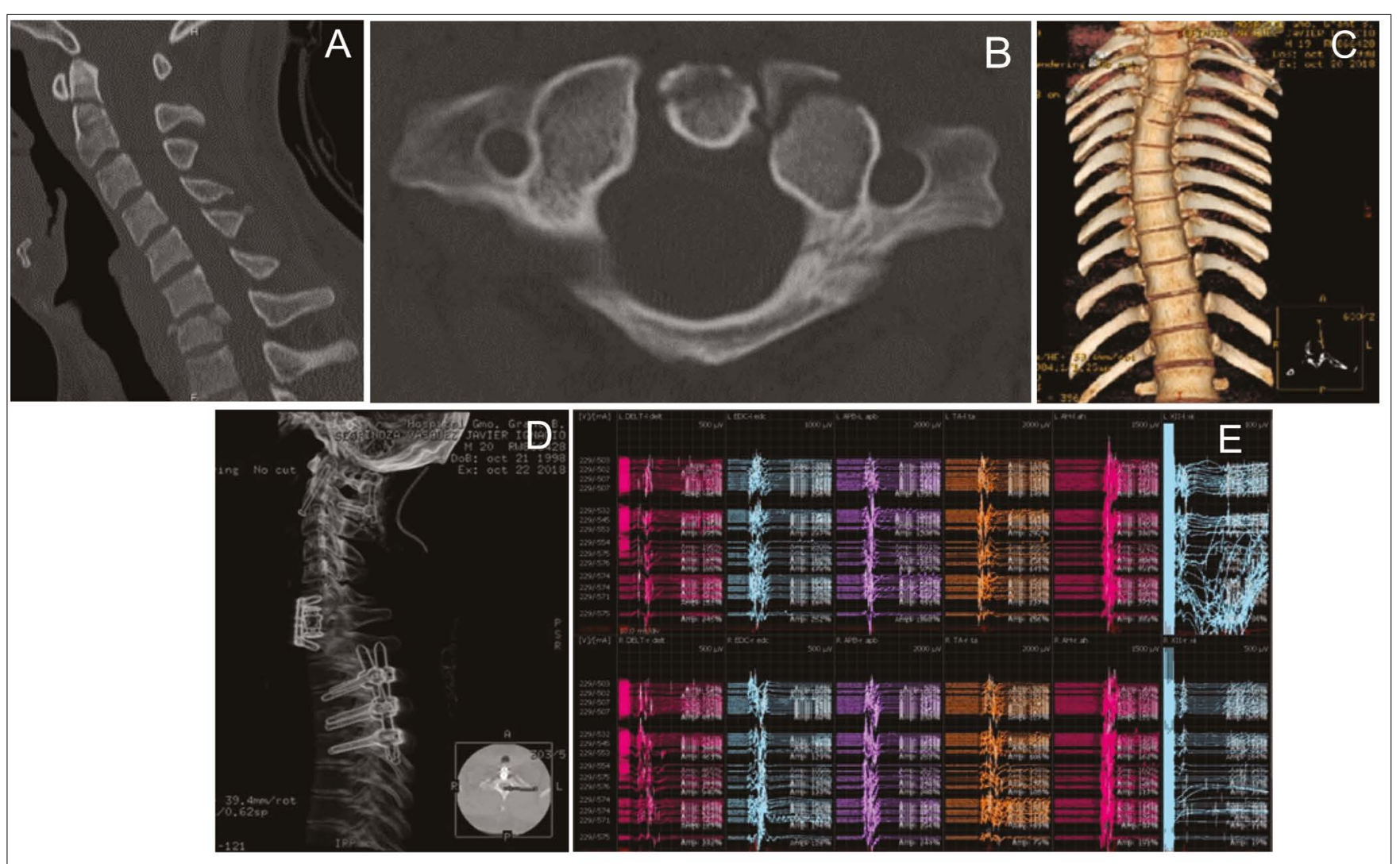

Figura 2. Caso Traumático. A) TC sagital preoperatorio evidenciando fractura de $\mathrm{C} 2$ y C7; B) TC corte axial en $\mathrm{C} 1$ que demuestra masa lateral flotante de $\mathrm{C} 1$ izq; C) TC en 3D que muestra fractura T4 en contexto escoliosis; D) Reconstrucción TC mostrando sistemas de osteosíntesis múltiples utilizados; E) Cascada de potenciales evocados motores del caso. 


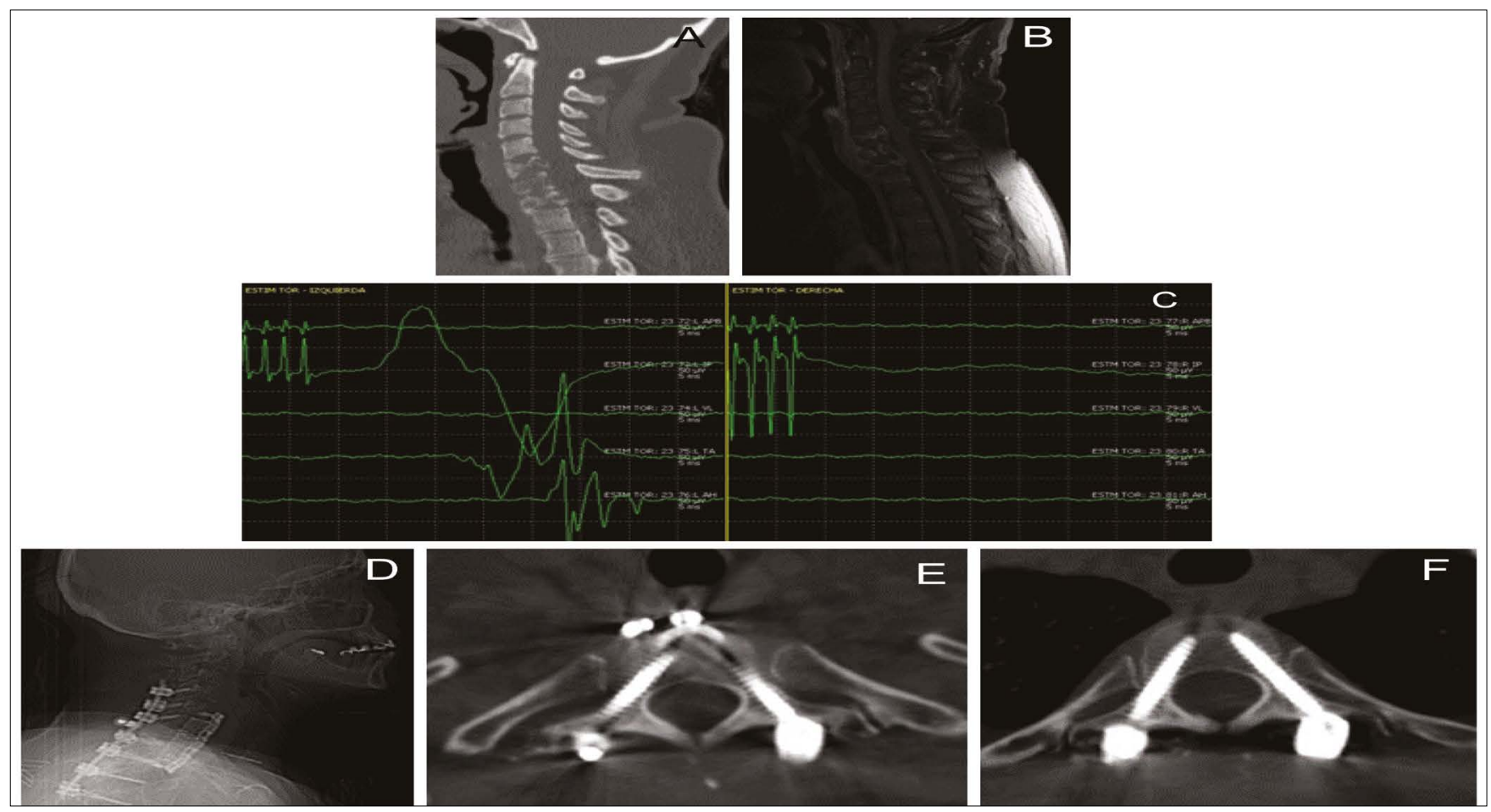

Figura 3. Caso Tumoral. A) TC preoperatorio; B) RNM preoperatoria; C) Estimulación tornillos transpediculares en umbral de seguridad; D) Radiografía evidenciando cosntructo $360^{\circ}$; E) TC axial con canastillo anterior y fijación transpedicular en T1; F) Tornillos transpediculares T2.

mente. Radiografía constructo 360 in situ (Figura 4D).

\section{Deformidad}

Paciente mujer de 50 años, portadora de enfermedad de Parkinson en tratamiento, antecedente de infarto cerebral hace 4 años, autovalente en actividades de la vida diaria. Dado cuadro de tetraparesia progresiva, se estudia con RNM columna cervical (Figura 5A) y TC (Figura 5B) que evidencian espondilolistesis C3-C4 grado II con mielopatía segmentaria, cifosis cervical C2-C7 de $45^{\circ}$, C2 tilt $>20^{\circ}$ y T1 slope $<30^{\circ}$. Columna lumbar con flat back, hipocifosis dorsal, SVA neutro. Dado lo anterior, se define corporectomía C4-C5 + placa anterior C3-C6 y vía posterior C3-C6 bajo MIO (Figura $5 \mathrm{C}$ ). Al reducir cifosis, potenciales lado der. caen muy significativamente (Figura 5D), y al disminuir distracción asociado a maniobras de reanimación, recupera progresivamente (Figura 5E). Evoluciona favorablemente y sin nuevo déficit, con reducción de listesis de 5 a $1 \mathrm{~mm}$ C3-C4, cifosis baja de $45^{\circ}$ a $25^{\circ}$, mejoría neurológica progresiva en controles postoperatorios.

\section{Discusión}

La mielopatía cervical compresiva es una enfermedad de columna progresiva, y la más común causa de disfunción de médula espinal en población añosa en el mundo ${ }^{5}$. Se caracteriza por compresión de médula espinal secundario a un estrechamiento en el diámetro sagital del canal espinal por cambios degenerativos en la columna cervical. Recientemente, se ha incrementado significativamente el uso de MIO para prevenir déficit neurológico durante cirugía de columna ${ }^{6}$, en que los cambios intraoperatorios obtenibles durante el MIO se corresponden con las funciones medulares durante la cirugía.

El rol del MIO comienza desde la posición quirúrgica en adelante, con obtención de línea de base que sirve de registro gráfico de presencia o ausencia de potenciales preoperatorios, herramienta médico-legal. En mejor escenario, la posición de la cabeza, columna y extremidades pueden verse severamente afectadas dado el desmedro de su función respecto a una línea de base en posición supino y neutro. En vías anteriores, la hiperextensión cervical, con o sin Jaquima, pudiera determinar caída de potenciales previo a la incisión? En vías posteriores, la hiperflexión puede ser determinante en un desmedro neurológico "no relacionado" a la cirugía misma. En prono, la sobreabducción de brazos o hipertracción de hombros, pudiera determinar plexopatías postural inferior o superior, respectivamente, además de neuropatías compresivas de nervio cubital entre otros. Todo lo anterior, prevenible en tiempo real relacionado solo a la posición quirúrgica.

Ocasionalmente y de acuerdo al tiempo de evolución, es posible observar mejoría funcional intraoperatoria. Algunos mecanismos pueden explicar la relación entre potenciales evocados motores transcraneales (TcMEP) y mejoría funcional, que en contexto de mielopatía, incluso se pudiera ver una mejoría posterior a la descompresión ${ }^{8}$, probablemente secundario a la mejoría en la excitabilidad neuronal del tracto corticoespinal, o mejoría enflujo sanguineo medular segmentario, o ambas. TcMEP representa el tracto corticoespinal descendiendo desde el área motora primaria al cuerno anterior de la médula espinal, seguido de un conjunto de raíces que tras un marcado entrecruzamiento, forman nervios periféricos que 


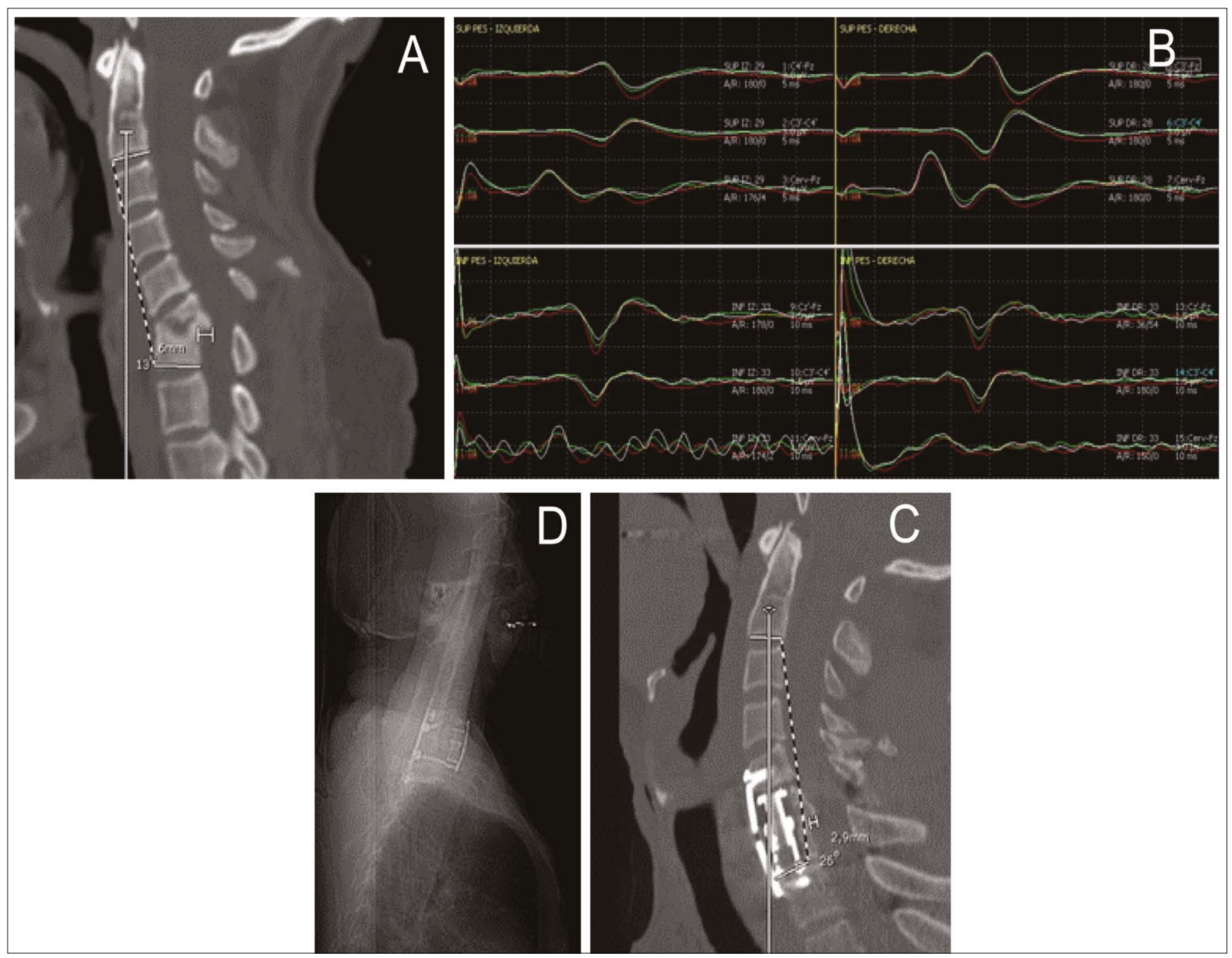

Figura 4. Caso Infeccioso. A) TC sagital con espondilodiscitis deformante C6-C7; B) Potenciales evocados somatosensoriales de 4 extremidades; C) TC postoperatorio con corrección de deformidad significativa; D) Radiografía de constructo $360^{\circ}$.

desembocan en los distintos músculos efectores. Tanto la mejoría como el deterioro funcional intraoperatorio de origen radicular pudieran estar afectados por este entrecruzamiento, pues en caso de no corresponder a una raíz dominante "lesionada" o "mejorada", son las raices adyacentes quienes están conduciendo esa infomación, no representando cambios significativos gráficos intraoperatorios ${ }^{9}$.

En base al último punto, se ha realizado mapeo heurístico de la inervación miotómica a través de estimulación radicular intraoperatoria directa, demostrando marcada variabilidad e inesperada participación muscular de parte de raíces "no contempladas" previamente. Comparada con la neuroanatomía clásica, un número significativo de raíces inervan un grupo mas amplio de músculos que lo esperado, dando cuenta de la gran variabilidad de presentación entre pacientes con el mismo número de raíces y localización ${ }^{10}$, a pesar de tratarse de plexo braquial, pues en plexo lumbosacro esta variabilidad es aún mayor.

Sin embargo, en la detección de daño de origen medular, que pudiera ser mas significativo a nivel de columna cervical durante cirugías complejas, los valores de TcMEP presentan alta confiabilidad en detectar la integridad funcional del tracto corticoespinal, con valores de sensibilidad y especificidad elevados ${ }^{11}$.

Inicialmente, se consideró latencia y amplitud de potenciales evocados somatosensoriales (SSEP) como evaluación de integridad medular, validado en 1995 en más de 50.000 casos de deformidades operados con $\mathrm{MIO}$, en que se deja establecido que esta herramienta disminuye significativamente el nuevo déficit neurológico ${ }^{12}$. Sin embargo, en el transcurso del tiempo se documentan casos en quienes a pesar de no presentar deterioro en SSEP, despiertan con nuevo déficit motor $^{13}$. Por lo anterior, nace el concepto de Monitoreo Neurofisiológico Multimodal, en que durante cirugías de columna compleja se suman las capacidades de cada modalidad en la detección de daño, lo que globalmente se torna altamente confiable.

Existe controversia respecto a los criterios de alarma en TCMEP. Tradicionalmente, se considera $10 \%$ aumento de latencia, pero a un $50 \%$ disminución de amplitud. Otros autores 


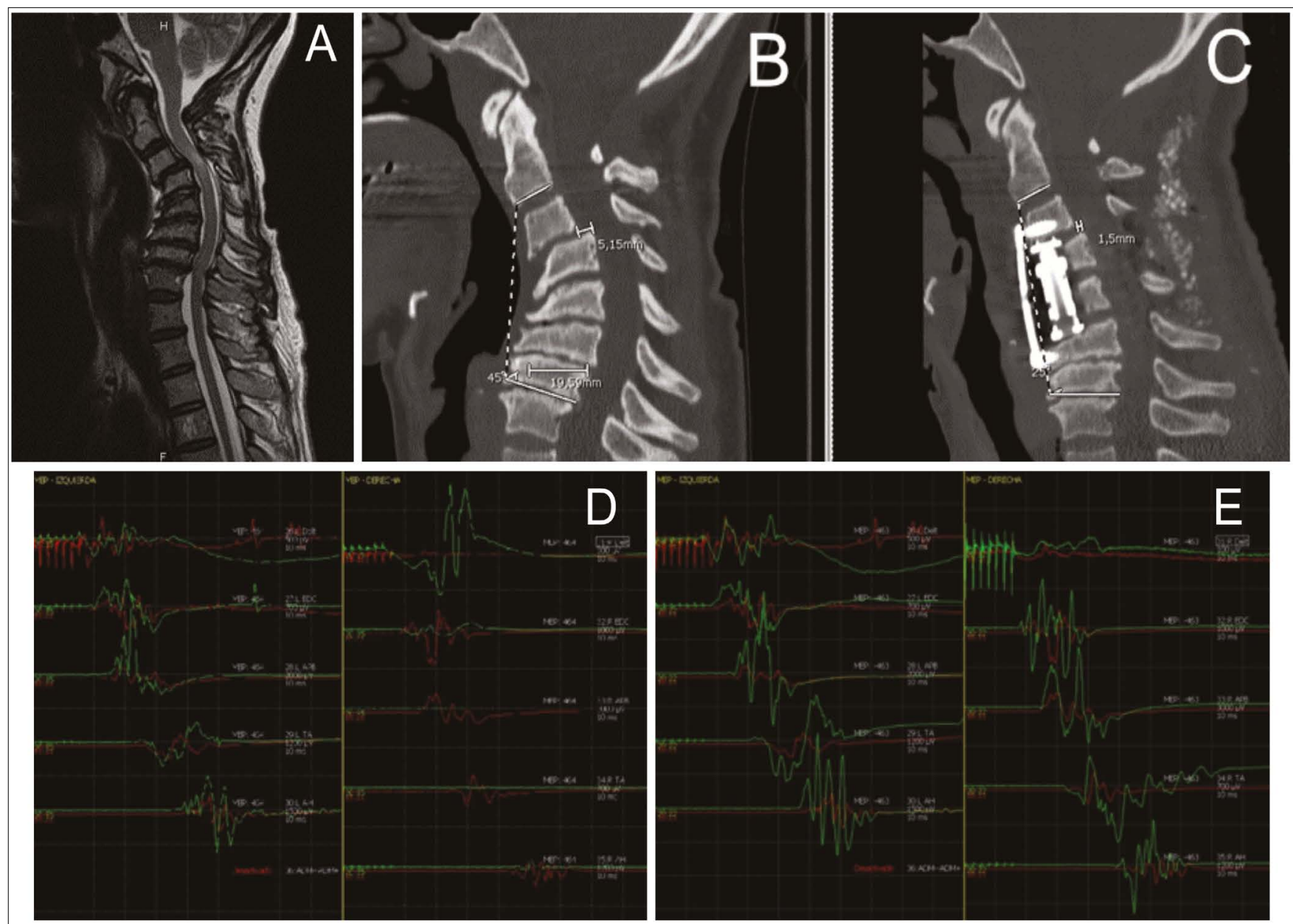

Figura 5. Caso deformidad. A) RNM preoperatoria con mielopatía C3-C4; B) TC preoperatorio con cifosis cervical y listesis C3-C4; C) TC postoperatorio con reducción subtotal de cifosis y listesis; D) Caída muy significativa potenciales motores lado der. En momento de reducción; E) Recuperación de potenciales al disminuir grado de reducción.

han considerado $80 \%$, mientras otros "todo o nada". Se ha dado importancia al número de fase, morfología, latencia, amplitud, incluso criterio multiparamétrico ${ }^{14}$. Cada metodología anterior cuenta con distinta capacidad de captar daño, muy en especial el que tendrá repercusión clínica. Nuestro criterio de alarma ha sido $50 \%$ de descenso amplitud focal, unilateral o bilateral, con o sin deterioro de potenciales somatosensoriales, y si han sido transitorios o permanentes durante el caso. Caídas significativas y permanentes se relacionan con mayor probabilidad a tener repercusión clínica postoperatoria.

Caída de potenciales SSEP o TCMEP o ambos es, probablemente, el evento intraoperatorio más devastador al que se enfrenta un cirujano de columna, pues la mortalidad intraoperatoria afortunadamente es excepcional. Existen maniobras de corrección intraoperatoria para que esta caída no llegue a tener expresión clínica, habiendo múltiples esquemas propuestos, pero siendo el "Protocolo TIP" el más reproductible y aplicable en momentos de extremo conflicto: T de Tiempo y Temperatura, deTener maniobras quirúrgicas, reTirar implantes que se relacionaron con la caída; I de Irrigación con suero fisiológico tibio y con papaverina al extradural o lecho; $P$ de Presión arterial que requiere ascender 15-20 puntos la media y Papaverina endovenosa en caso de no existir contraindicación. Con todo lo anterior, existe recuperación parcial o total de potenciales en forma progresiva, y cuando no, el déficit instaurado pasa a ser transitorio en vez de permanente ${ }^{15}$.

Relacionado a lo anterior, dentro de esta serie se analizó previamente los vía posterior, quienes en principio se realizaba descompresiva sin instrumentación para estenoraquis degenerativa multinivel o Sd. Centromedular traumático, evidenciando un significativo mayor deterioro comparativo a quienes se instrumentaron. Además, se evidenció que aquellos pacientes instrumentados y posteriormente descomprimidos presentaron menor caída de potenciales y sin repercusión clínica comparada con descompresión inicial e instrumentación a continuación ${ }^{16}$. Como mecanismo de caída potenciales pudiera ser que la microinestabilidad segmentaria, y en contexto de estenorraquis severa a crítica, asociado a la suplencia microvascular crónicamente enferma determinen un conflicto vascular global con la consecuente isquemia medular segmentaria y profundización del deterioro en mielopatía pre-existente, visualizable en caída TcMEP a pesar de línea de base "pobre", y con SSEP distales a sitio lesional sin cambios dada la habitual no conducción en este contexto. 
Utilización de nervio laríngeo recurrente en vía cervical anterior posee tasa de falsos (+) y falsos (-) no despreciable, costo elevado insumo (tubo endotraqueal con electrodos) y desbalance costo-beneficio. Hasta un $80 \%$ de los electrodos de las cuerdas vocales pudiera quedan con malposición, tanto craneo-caudal como rotacional, deteminando una incorrecta evaluación ${ }^{17}$. Existe mayor riesgo de daño en niveles cervicales mas bajos y cuando se realiza corporectomía, muchas veces este daño es subdiagnosticado. En casos complejos, sin bien forma parte integral de la multimodalidad, descentra la atención de lo esencial o de lo que pudiera tener mayor impacto funcional.

Estimulación de tornillos a masas laterales: punto controversial y con marcada desinformación comparativa respecto a columna lumbar en la literatura. Última evidencia disponible apunta a distancia de tornillos hacia canal menor a $2 \mathrm{~mm}$ con umbral de corte $7,5 \mathrm{~mA}$, con $75 \%$ sensibilidad y $92 \%$ especificidad $^{18}$. Nuestros tornillos fueron todos estimulados en principio, sin embargo, comenzamos a percatar altos falsos $(+)$, esto es, umbral percibido demostraba cercanía o contacto con estructuras neurales y TC postoperatorio además de cuadro clínico no eran compatibles. Comparativamente a la muy escasa evidencia disponible, la diferencia pudo radicar en el tiempo de estimulaciòn utilizado, de 50 usec a $3.1 \mathrm{~Hz}$ contra 200-300 usec a 2-3 Hz, lo que pudiera estar facilitando enormemente evocar respuestas de musculatura plexo braquial y ser captados como falsos (+). Falsos (-) son excepcionales.

Estimulación de tornillos torácicos altos para fijaciones de charnela cervico-torácica: punto en extremo controversial. Tradicionalmente, se ha utilizado estimulación tornillo transpedicular y registro en musculatura intercostal o abdominal, sin embargo, con tasa de falsos (+) y (-) del orden del $50 \%$, que lo consideramos inaceptable desde el inicio. Gema de Blas desarrolla una técnica de registro para columna torácica alta, T2-T6, con registro a altura de línea media axilar que mejora resultados, aunque persisten muy optimizables: $36 \%$ de malposición de tornillos a pesar de umbral correcto ${ }^{19}$. Blair Calancie marca algunas dieferencias sustanciales al respecto, con estimulación trayecto pedicular (y no alma tornillo), estimulación técnica multipulso y no estímulo simple como para columna cervical y lumbar, y registro en musculatura de extremidades inferiores en vez de intercostal y abdominal. Lo anterior se publica $2014^{20}$, y nuestro grupo lo presenta el 2015 con los 250 primeros tornillos torácicos con esta técni$\mathrm{ca}^{21}$, persistiendo aún como una marcada controversia mundial respecto al mecanismo de obtención de la respuesta al existir malposición medial: Reflejo $\mathrm{H}$ o despolarización directa del tracto corticoespinal. Interrelacionando lo anterior a la técnica quirúrgica, tornillos T1-T2 en pacientes con T1 slope elevado, tendieron a obtener respuesta del músculo abductor pollicis brevis y no extremidades inferiores, que cuando no fue considerada patológica, se demostró malposición superior a plataforma (no foraminal) o punta del tornillo externa, que se interpreta como despolarización C8-T1.

En conclusión, el MIO en columna cervical compleja entrega información y seguridad no evaluables a través de ninguna otra herramienta disponible en Neurocirugía, determinando cambios quirúrgicos intraoperatorios y como curva de aprendizaje que favorecen dramáticamente los resultados. Considero muy fuertemente, que el MIO debe formar parte del armamentario actual de herramientas disponibles, para el desarrollo correcto de la Neurocirugía en Chile, en un intento incansable de ofrecer a nuestro paciente neuroquirúrgico un tratamiento de clase mundial.

\section{Referencias}

1. Baabor M, Cruz S, Villalón J. Actualización en la fisiopatología y manejo de traumatismo raquimedular. Revisión bibliográfica. Rev. Chil. Neurocirugía 42: 144-150, 2016.

2. Khorasanizadeh M, Yousefifard M, Eskian M, Lu Y, Chalangari M, Harrop JS, Jazayeri SB, Seyedpour S, Khodaei B, Hosseini M, Rahimi-Movaghar V. Neurological recovery following traumatic spinal cord injury: a systematic review and meta-analysis. J Neurosurg Spine. 2019, Feb 15:1-17.

3. Joaquim AF, Makhni MC, Riew KD. Post-operative nerve injuries after cervical spine surgery. Int Orthop. 2019 Apr;43(4):791-795.

4. Costa P, Faccani G, Sala F, Montalenti E, Giobbe ML, Deletis V. (2014). Neurophysiological assessment of the injured spinal cord: an intraoperative approach. Spinal Cord, 52(10), 749-757.

5. Fehlings MG, Tetreault LA, Riew KD, Middleton JW, Wang JC. A clinical practice guideline for the management of degenerative cervical myelopathy: introduction, rationale, and scope. Global Spine J. 2017;7(3 Suppl):21S-27S.

6. Devlin VJ, Schwartz DM. Intraoperative neurophysiologic monitoring during spinal surgery. J Am Acad Orthop Surg. 2007;15(9):549-560.

7. Faisal R, Jahangiri, Andrea Flolmberg, Francisco Vega-Bermudez \& Vincent Arlet (2011). Preventing Position-Related Brachial Plexus Injury with Intraoperative Somatosensory Evoked Potentials and Transcranial Electrical Motor Evoked Potentials during Anterior Cervical Spine Surgery, American Journal of Electroneurodiagnostic Technology, 51:3, 198-205.

8. Wang S, Tian Y, Wang C, et al. Prognostic value of intraoperative MEP signal improvement during surgical treatment of cervical compressive myelopathy. Eur S J. 2016;25(6):1875-1880.

9. Appel S, Biron T, Goldstein K, Ashkenazi E. (2018). The effect of intra and extra surgical factors on the efficacy of intraoperative neuromonitoring during cervical spine surgery. World Neurosurgery.

10. Schirmer CM, Shils JL, Arle JE, Cosgrove GR, Dempsey PK, Tarlov E, et al. (2011). Heuristic map of myotomal innervation in humans using direct intraoperative nerve root stimulation. Journal of Neurosurgery: Spine, 15(1), 64-70.

11. Hilibrand AS, Schwartz DM, Sethuraman V, Vaccaro AR, Albert TJ. Comparison of transcranial electric motor and somatosensory evoked potential monitoring during cervical spine surgery. $J$ Bone Joint Surg Am. 2004;86-A(6):1248-1253.

12. Nuwer MR, Dawson EG, Carlson LG, Kanim LE, Sherman JE. Somatosensory evoked potential spinal cord monitoring reduces neurologic deficits after scoliosis surgery: results of a large multicenter survey. Electroencephalogr Clin Neurophysiol. 1995 Jan;96(1):6-11.

13. García RM, Qureshi SA, Cassinelli EH, Biro CL, Furey CG, Bohlman $\mathrm{HH}$. Detection of postoperative neurologic deficits using somatosensory evoked potentials alone during posterior cervical laminoplasty. Spine J. 2010 Oct;10(10):890-5.

14. MacDonald DB. Overview on Criteria for MEP Monitoring. J Clin Neurophysiol. 2017 Jan;34(1):4-11. 
15. Cabrera Cousiño JP, Luna F, Torche E, Vigueras S, Torche M, Condal M, et al. Utility of "TIP Protocol" in Complex Spine Surgery: An Effective, Simplified and Reproducible Rescue Algorithm of Management when Intraoperative Neurophysiological Potentials Fall Down. Global Spine Congress, May 2019, Toronto, Canada.

16. Cabrera JP, Luna F, Torche E, Rivas W, Torche M, Vigueras $S$, et at. Posterior Subaxial Cervical and Cervicothoracic Joint Spine Fixations: Recommendations from an Intraoperative Neurophysiological Monitoring Point of View. Global Spine Congress, May 2018, Singapore.

17. Sinclair CF, Téllez MJ, Tapia OR, Ulkatan S, Deletis V. (2017). A novel methodology for assessing laryngeal and vagus nerve integrity in patients under general anesthesia. Clinical Neurophysiology, 128(7), 1399-1405.

18. Wilson B, Curtis E, Hirshman B, Oygar A, Chen K, Gabel BC, et al. (2017). Lateral mass screw stimulation thresholds in posterior cervical instrumentation surgery: a predictor of medial deviation. Journal of Neurosurgery: Spine, 26(3), 346-352.

19. Regidor I, de Blas G, Barrios C, Burgos J, Montes E, GarcíaUrquiza S, Hevia E. (2011). Recording triggered EMG thresholds from axillary chest wall electrodes: a new refined technique for accurate upper thoracic (T2-T6) pedicle screw placement. European Spine Journal, 20(10), 1620-1625.

20. Calancie B, Donohue ML, Harris CB, Canute GW, Singla A, Wilcoxen KG, Moquin RR. (2014). Neuromonitoring with pulse-train stimulation for implantation of thoracic pedicle screws: a blinded and randomized clinical study. Part 1 . Methods and alarm criteria. Journal of Neurosurgery: Spine, 20(6), 675-691.

21. Cabrera Cousiño JP, García R, Lastra L, Vigueras S, Torche Vélez M. (2015). Assessment of Medial Malpositioning during Thoracic Pedicle Screws Placement with a Contemporary Intraoperative Neurophysiological Monitoring Method. Global Spine Journal, 5(1_suppl), s-0035-1554449-s-0035-1554449. 